\title{
SSSPM J1102-3431 brown dwarf characterization from accurate proper motion and trigonometric parallax ${ }^{\star}$
}

\author{
R. Teixeira ${ }^{1,2}$, C. Ducourant ${ }^{2,1}$, G. Chauvin ${ }^{3}$, A. Krone-Martins ${ }^{1,2}$, I. Song ${ }^{4}$, and B. Zuckerman ${ }^{5}$ \\ 1 Instituto de Astronomia, Geofísica e Ciências Atmosféricas, Universidade de São Paulo, Rua do Matão, 1226 - Cidade Universitária, \\ 05508-900 São Paulo SP, Brazil \\ e-mail: teixeira@astro.iag.usp.br \\ 2 Observatoire Aquitain des Sciences de l'Univers, CNRS-UMR 5804, BP 89, 33270 Floirac, France \\ ${ }^{3}$ Laboratoire d'Astrophysique, Observatoire de Grenoble, 414 rue de la piscine, 38400 Saint-Martin d'Hères, France \\ ${ }^{4}$ Spitzer Science Center, IPAC/Caltech, MS 220-6, Pasadena, CA 91125, USA \\ 5 Department of Physics \& Astronomy and Center for Astrobiology, University of California, Los Angeles, Box 951562 , \\ CA 90095, USA
}

Received 5 May 2008 / Accepted 13 June 2008

\begin{abstract}
Context. In 2005, Scholz and collaborators discovered, in a proper motion survey, a young brown dwarf SSSPM J1102-3431 (SSSPM J1102) of spectral type M8.5, probable member of the TW Hydrae Association and possible companion of the T Tauri star TW Hya. The physical characterization of SSSPM J1102 was based on the hypothesis that it forms a binary system with TW Hya. The recent discovery of a probable giant planet with a very short-period inside the TW Hya protoplanetary disk, as well as a disk around SSSPM J1102, make it especially interesting and important to measure well the physical parameters of SSSPM J1102. Aims. Trigonometric parallax and proper motion measurements of SSSPM J1102 are necessary to test for TWA membership and, thus, to determine the mass and age of this young brown dwarf and the possibility that it forms a wide binary system with TW Hya. Methods. Two years of regular observations at the ESO NTT/SUSI2 telescope have enabled us to determine the trigonometric parallax and proper motion of SSSPM J1102.

Results. With our accurate distance determination of $55.2_{-1.4}^{+1.6} \mathrm{pc}$ and proper motions of $(-67.2,-14.0) \pm 0.6 \mathrm{mas} / \mathrm{yr}$, we could confirm SSSPM J1102 as a very probable member of TWA. Assuming the TW Hydrae association age of 5-10 Myr, the evolutionary models compared to the photometry of this young brown dwarf indicate a mass of $M=25 \pm 5 M_{\text {Jup }}$ and an effective temperature $T_{\text {eff }}=2550 \pm$ $100 \mathrm{~K}$.

Conclusions. Our parallax and proper motion determination allow us to precisely describe the physical properties of this low mass object and to confirm its TWA membership. Our results indicate that SSSPMJ1102 may be a very wide separation companion of the star TW Hya.
\end{abstract}

Key words. stars: low-mass, brown dwarfs - astrometry - stars: distances - stars: fundamental parameters Galaxy: open clusters and associations: individual: TW Hya

\section{Introduction}

The TW Hydrae Association (TWA) is a young, nearby association consisting of about 25 known members. Due to its youth and proximity, this association has been intensively studied in the last decade revealing a great variety of systems: tight astrometric binaries well-suited to calibration of PMS models; stars and brown dwarfs harboring circumstellar disks, planetary and brown dwarf companions; and more recently a putative massive planet embedded in its own proto-planetary disks (TW Hya; Setiawan et al. 2008). Surprisingly, only five members have known trigonometric parallaxes. De la Reza et al. (2006) report a trace-back age of $8.3 \pm 0.8 \mathrm{Myr}$, independent of evolutionary models. Relying on astrometric and spectroscopic data, the Galactic space motions of TWA members are traced backward in time until they occupy a minimum volume in space. This age estimation would greatly benefit from parallax measurements of additional TWA members.

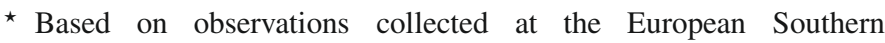
Observatory, Chile (76.C-0543, 077.C-0112, 078.C-0158, 079.C-0229) and at Valinhos meridian circle.
Scholz et al. (2005) discovered a new young sub-stellar object, SSSPM J1102-3431 (SSSPM J1102), a probable member of TWA. Its photometric and spectroscopic characteristics suggest a young brown dwarf of spectral type M8.5. Located 12' from TW Hya and sharing similar proper motions, Scholz et al. (2005) suggested that SSSPM J1102 could form a binary system with TW Hya. Assuming an age of 10 Myr (Webb et al. 1999) and the Hipparcos distance for TW Hya, they derived for SSSPM J1102 a mass of $\approx 25 M_{\text {Jup }}$. Recently, a flat opticallythick disk was discovered around SSSPM J1102 (Riaz \& Gizis 2008) based on a reconstructed mid-infrared spectral energy distribution using broadband photometry (Sterzik et al. 2004; Riaz et al. 2006). Utilizing combined NASA, IRTF, and Spitzer spectroscopic observations, Morrow et al. (2008) argued in favor of high degrees of dust settling to the disk midplane as well as significant grain growth in the upper layers, suggesting rapid dust processing compared to disks around stars.

Characterization of SSSPM J1102 itself and its disk properties and the question of binarity status with TW Hya make a distance determination of substantial interest. Since January 2006 we have conducted astrometric and photometric observations 
Table 1. Astrometric parameters for SSSPM J1102-3431 derived in this work. Proper motions and parallax are absolute quantities.

\begin{tabular}{cccc}
\hline \hline$\mu_{\alpha_{\mathrm{abs}}}^{*}(\mathrm{mas} / \mathrm{yr})$ & $\mu_{\delta_{\mathrm{abs}}}(\mathrm{mas} / \mathrm{yr})$ & $\pi_{\mathrm{abs}}(\mathrm{mas})$ & $d(\mathrm{pc})$ \\
\hline$-67.2 \pm 0.6$ & $-14.0 \pm 0.6$ & $18.1 \pm 0.5$ & $55.2_{-1.4}^{+1.6}$ \\
\hline
\end{tabular}

from the New Technology Telescope at the European Southern Observatory (ESO NTT telescope) to derive the trignometric parallax of SSSPM J1102. Our observations are presented in Sect. 2. The data reduction and analysis and the result of this trigonometric parallax programme are given in Sect. 3. Finally, membership in TWA, the physical properties of SSSPM J1102 compared to other TWA substellar objects, and the binarity status with TW Hya are discussed, respectively, in Sects. 4-6.

\section{Observations}

Astrometric and photometric $(V, R, I)$ observations were performed between 2006 and 2007 in direct imaging mode with the ESO NTT-SUSI 2 instrument, which ensures a good compromise between a large field of view $\left(5.5^{\prime} \times 5.5^{\prime}\right)$ for a sufficient sample of background stars and a small pixel size (80.5 mas) necessary to reach sub-milli arcsecond astrometric precision.

Data at six observational epochs were acquired with a total of twelve nights of observation. All observations were done around transit to minimize the differential color refraction effects (DCR). Multiple exposures were obtained at each epoch to reduce astrometric errors and enhance $\mathrm{S} / \mathrm{N}$. An $I$-band filter was selected to minimize the DCR effect. Residual DCR effects were removed from single observations, following methods described in Ducourant et al. (2007).

The alignment of CCD axes and the scale determination were obtained using the 2MASS catalog (Cutri et al. 2003) and from CCD meridian observations at Valinhos Observatory (Teixeira et al. 2000).

All image frames were measured using the DAOPHOT II package (Stetson 1987), fitting a stellar point-spread function for each frame. Finally, we created catalogs of measured positions $(x, y)$, internal magnitudes, and associated errors for all stars on each frame.

\section{Trigonometric parallax determination}

These observational data were processed through a global treatment as described in Ducourant et al. (2007) and Ducourant et al. (2008), and a solution was derived for SSSPM J1102, relative to background stars $(I \leq 22.5 \mathrm{mag})$ : $\pi_{\text {rel }}=17.5 \pm 0.6 \mathrm{mas}$, $\mu_{\alpha_{\text {rel }}}^{*}=-61.0 \pm 0.6 \mathrm{mas} / \mathrm{yr}$, and $\mu_{\delta_{\text {rel }}}=-15.0 \pm 0.6 \mathrm{mas} / \mathrm{yr}$. Then, a statistical conversion from relative to absolute parallax and proper motions, based on the Besançon Galaxy model (Robin et al. 2003; Robin et al. 2004), was derived ( $\Delta \pi=0.6$ mas, $\Delta \mu_{\alpha}^{*}=-6.2 \mathrm{mas} / \mathrm{yr}, \Delta \mu_{\delta}=+1.0 \mathrm{mas} / \mathrm{yr}$ ). In Table 1 , we give absolute astrometric parameters and, in Table 2 the photometry for SSSPM J1102 obtained in this work.

We present in Fig. 1 the observations of SSSPM J1102, together with the fitted path (relative parallax and proper motions).

\section{Membership in TWA}

The distance derived in this work $\left(d=55.2_{-1.4}^{+1.6} \mathrm{pc}\right)$ is well within the limits of the boundaries of the photometrically-derived distances of the 25 TWA members
Table 2. Bessel ( $V, R$, and $I$ derived in this work) and 2MASS ( $J$, $H$ and $K$, Cutri et al. 2003) apparent and absolute magnitudes for SSSPM J1102-3431.

\begin{tabular}{crr}
\hline \hline & \multicolumn{1}{c}{$m(\mathrm{mag})$} & \multicolumn{1}{c}{$M(\mathrm{mag})$} \\
\hline$V$ & $21.46 \pm 0.04$ & $17.75 \pm 0.19$ \\
$R$ & $19.14 \pm 0.04$ & $15.43 \pm 0.19$ \\
$I$ & $17.90 \pm 0.03$ & $14.19 \pm 0.18$ \\
$J$ & $13.034 \pm 0.024$ & $9.32 \pm 0.17$ \\
$H$ & $12.356 \pm 0.022$ & $8.65 \pm 0.17$ \\
$K$ & $11.887 \pm 0.024$ & $8.18 \pm 0.17$ \\
\hline
\end{tabular}

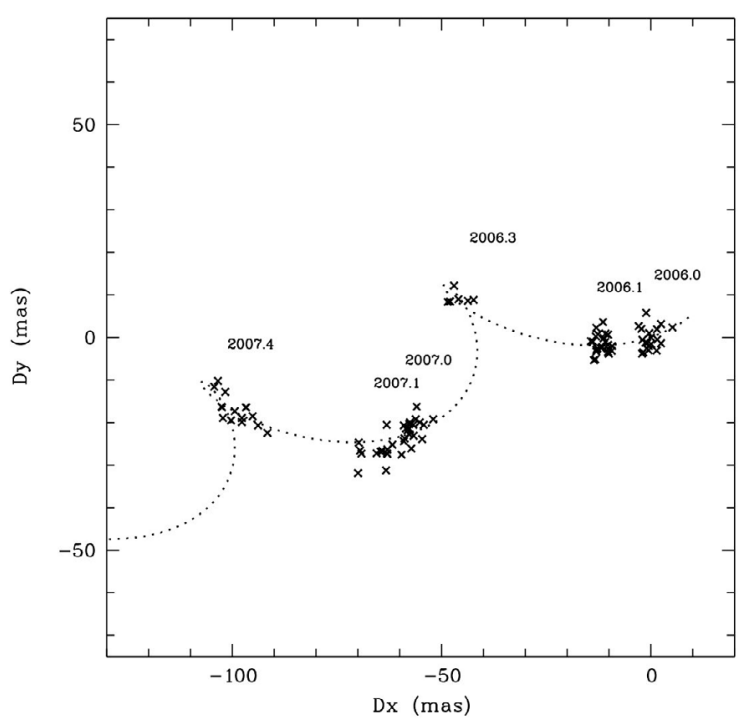

Fig. 1. Observations together with the fitted path due to parallax and proper motion for SSSPM J1102. The dashed curve represents the least squares solution.

(Zuckerman \& Song 2004): 20 pc to 130 pc and also in good accordance with the boundaries of the accurate HIPPARCOS parallaxes (ESA 1997) of 5 members: 47 to 104 pc.

The proper motions obtained in this work agree, at about the $1 \sigma$ level, with those obtained by Scholz et al. (2005) $(-82 \pm$ $12 \mathrm{mas} / \mathrm{yr},-12 \pm 6 \mathrm{mas} / \mathrm{yr})$ and are fully compatible with the proper motions of TWA members ( $\mu_{\alpha}^{*}$ from -33 to -122 mas/yr, $\mu_{\delta}$ from -10 to -43 mas/yr).

Following the de Bruijne (1999) convergent point method, we can test the membership of SSSPM J1102 in TWA. Using our proper motion results, and the estimate of TWA's convergent point obtained by Mamajek (2005) $(\alpha=103.2 \pm 1.5$, $\delta=-30^{\circ} .7 \pm 1.5$ ), we find that most of SSSPM J1102 proper motion's magnitude is concentrated in the direction toward the convergent point $\left(\mu_{\|}=-68.55 \pm 0.61 \mathrm{mas} \mathrm{yr}^{-1}\right)$ and that its perpendicular component is small $\left(\mu_{\perp}=3.06 \pm 2.32\right.$ mas yr$\left.^{-1}\right)$, being compatible with zero, as expected for an ideal cluster member. The accordance of both our distance estimate and our proper motions with the characteristics of TWA strongly supports the membership of SSSPM J1102 in the TWA Association.

\section{Physical properties}

Considering SSSPM J1102 as a member of TWA, one can use evolutionary model predictions to derive the physical properties of this young M8.5 brown dwarf. We compared its 2MASS JHK photometry (converted into the CIT system) with the predictions of the DUSTY (Chabrier et al. 2000) evolutionary model for a given age of 5-10 Myr. The predicted mass, 
Table 3. Physical properties of SSSPM J1102 compared to the properties of the five other substellar systems in TWA. Photometric distances in parenthesis.

\begin{tabular}{llllll}
\hline \hline Name & $\begin{array}{l}d \\
(\mathrm{pc})\end{array}$ & SpT & $\begin{array}{l}\text { Mass } \\
\left(M_{\text {Jup }}\right)\end{array}$ & $\begin{array}{l}T_{\text {eff }} \\
(\mathrm{K})\end{array}$ & $\log \left(L / L_{\odot}\right)$ \\
\hline 2M1207 A & 52.4 & M8 & $25 \pm 5$ & $2550 \pm 150$ & $-2.7 \pm 0.2$ \\
2M1139 & $(46)$ & M8 & $25 \pm 5$ & $2550 \pm 150$ & $-2.6 \pm 0.3$ \\
TWA5 B & $(45)$ & M8.5 & $25 \pm 5$ & $2550 \pm 150$ & $-2.6 \pm 0.3$ \\
SSSPM J1102 & 55.2 & M8.5 & $25 \pm 5$ & $2550 \pm 150$ & $-2.6 \pm 0.3$ \\
DENIS 1245 & $(90)$ & M9.5 & $15-20$ & $2350 \pm 150$ & $-2.9 \pm 0.3$ \\
2M1207 B & 52.4 & late-L & $8 \pm 2$ & $1600 \pm 100$ & $-3.8 \pm 0.1$ \\
& 52.4 & late-L & $4 \pm 1$ & $1150 \pm 150$ & $-4.5 \pm 0.2$ \\
\hline
\end{tabular}

effective temperature, surface gravity, and luminosity are reported in Table 3. They are compared to the (model-dependent) physical properties of the five other substellar dwarf objects of TWA, covering spectral types between M8 to mid-L and part of a large variety of systems: isolated (2M1139, Gizis 2002; DENIS 1245, Looper et al. 2007), in a binary (2M1207 AB, Chauvin et al. 2004; Chauvin et al. 2005) or as a companion to a stellar primary (TWA5 B, Lowrance et al. 1999). Uncertainties in the mass of 2M1207 B are discussed by Mohanty et al. (2007), Gizis et al. (2007), Mamajek \& Meyer (2007) and Ducourant et al. (2007). Distances (sometimes proper motion) remain uncertain for TWA5, 2M1139 and DENIS 1245 and would benefit from future parallax measurements. In this comparison we used the same model, the same age, and trigonometric or photometric (in parenthesis) parallaxes. In the case of 2M1207 B, the COND (Baraffe et al. 2003) model predictions are also considered, as discussed in Ducourant et al. (2007).

\section{Binarity}

Scholz et al. (2005) suggested that SSSPM J1102 forms a wide binary system with TW Hya, but with the lack of distance and accurate proper motion for SSSPM J1102, the authors could not draw any firm conclusions. The distance and proper motions derived here for SSSPM J1102 $\left(d=55.2_{-1.4}^{+1.6} \mathrm{pc},(-67.2,-14.0) \pm\right.$ $0.6 \mathrm{mas} / \mathrm{yr}$ ) can be compared with the recent "Hipparcos new reduction" catalog (Van Leeuwen 2007a,b) data for TW Hya ( $\pi=$ $22.12 \pm 3.08 \mathrm{mas},-68.0 \pm 2.8 \mathrm{mas} / \mathrm{yr},-15.1 \pm 2.2 \mathrm{mas} / \mathrm{yr})$. We observe that the two objects have similar proper motions but, perhaps, discrepant parallaxes. Additional parallax data will be required for TW Hya to establish whether the two objects could be a bound pair.

Considering SSSPM J1102 as a member of TWA, from our astrometric measurements and the convergent point from Mamajek 2005, we can estimate the SSSPM J1102 radial velocity $V_{\mathrm{r}}=14.13 \pm 0.79 \mathrm{~km} \mathrm{~s}^{-1}$ leading to the spacial velocity components: $(-11.4,-19.3,-4.7) \mathrm{km} \mathrm{s}^{-1}$, very similar to that of TW Hya's $(-11.7,-17.9,-4.9) \mathrm{km} \mathrm{s}^{-1}$.

From the coordinates determined here for SSSPM J1102 $\left(11^{\mathrm{h}} 02^{\mathrm{m}} 09.803^{\mathrm{s}},-34^{\circ} 30^{\prime} 35.56^{\prime \prime}, 2006.653\right)$ and the position and proper motions given in the new reduced Hipparcos for TW Hya $\left(11^{\mathrm{h}} 01^{\mathrm{m}} 51.954^{\mathrm{s}},-34^{\circ} 42^{\prime} 16.91^{\prime \prime}, 1991.25\right)$, we derive an angular separation in 2006.653 of $12.3^{\prime}$. From this angular separation and the distance of SSSPM J1102 (55.2 $2_{-1.4}^{+1.6} \mathrm{pc}$, this work), we derive a projected separation on the sky of about $41000 \mathrm{AU}$.

The good agreement between the kinematic characteristics of both objects shows that, in addition to occupying similar positions in space, they clearly share the same spatial movement.
However, the large separation does not support the hypothesis that these two objects are gravitationally bound. Complementary observations are necessary to clarify this point.

\section{Conclusions}

Motivated by the need to have accurate distance determinations for members of the TW Hydrae Association and by specific interest in the brown dwarf SSSPM J1102, we measured its trigonometric parallax with an error $<3 \%$, thus ensuring a precise distance determination and a refined physical characterization of this sub-stellar object. The good accordance of our precise distance and proper motions of SSSPM J1102 with those characterizing TWA ensure that this object belongs to the TWA Association.

Our results show that SSSPJ1102 and TW Hya are located in the same region of the space, and share similar Galactic space motions, but it is still not possible to decide if the two objects constitute a wide binary system as proposed by Scholz et al. (2005).

Acknowledgements. We would like to thank the staff of ESO-VLT and CFHT and Gilles Chabrier, Isabelle Baraffe, and France Allard for providing the latest update of their evolutionary models. We also acknowledge partial financial support from the Programmes Nationaux de Planétologie et de Physique Stellaire (PNP \& PNPS) (in France), the Brazilian Organism FAPESP and CAPES, and French Organism COFECUB.

\section{References}

Baraffe, I., Chabrier, G., Basmah, T. S., Allard, F., \& Hauschildt, P. H. 2003, A\&A, 402, 701

de Bruijne, J. H. J. 1999, MNRAS, 306, 381

Chabrier, G., Baraffe, I., Allard, F., \& Hauschildt, P. H. 2000, ApJ, 542, 464

Chauvin, G., Lagrange, A. M., Dumas, C., et al. 2004, A\&A, 425, L29

Chauvin, G., Lagrange, A. M., Dumas, C., et al. 2005, A\&A, 438, L25

Cutri, R. M., Skrustskie, M. F., Van Dyk, S., et al. 2003, 2MASS All Sky Catalog of Point Sources (Pasadena: IPAC/Caltech)

De la Reza, R., Jilinski, E., \& Ortega, V. G. 2006, AJ, 131, 2609

Ducourant, C., Teixeira, R., Hambly, N., et al. 2007, A\&A, 470, 387

Ducourant, C., Teixeira, R., Chauvin, G., et al. 2008, A\&A, 477, L1

ESA 1997, HIPPARCOS and Tycho catalogues, ESA SP-1200

Fabrycky, D., \& Tremaine, S. 2007, ApJ, 669, 1298

Gizis, J. E. 2002, ApJ, 575, 484

Gizis, J., Jao, W., Subsavage, J. P., \& Henry, T. J. 2007, ApJ, 669, L45

Looper, D. L., Burgasser, A. J., Kirkpatrick, J. D., \& Swift, B. J. 2007, ApJ, 669, L97

Lowrance, P. J., McCarthy, C., Becklin, E. E., et al. 1999, ApJ, 512, L69

Mamajek, E. 2005, ApJ, 634, 1385

Mamajek, E., \& Meyer, M. 2007 [arXiv:0709.0456M]

Mohanty, S., Jayawardhana, R., Huélamo, N., \& Mamajek, E. 2007, ApJ, 657, 1064

Morrow, A. L., Luhman, K. L., Espaillat, C., et al. 2008, ApJ, 676, L143

Riaz, B., \& Gizis, J. E. 2008 [arXiv:0802 . 2048]

Riaz, B., Gizis, J. E., \& Hmiel, A. 2006, 639, L79

Robin, A. C., Reyle, C., Derriere, S., \& Picaud, S. 2003, A\&A, 409, 523

Robin, A. C., Reyle, C., Derriere, S., \& Picaud, S. 2004, A\&A, 416, 157

Scholz, R. D., McCaughream, M. J., Zinnecker, H., \& Lodieu, N. 2005, A\&A, 430, L49

Setiawan, J., Henning, Th., Launhardt, R., et al. 2008, Nature, 451, 7174, 38 Sterzik, M. F., Pascucci, I., Apai, D., van der Bliek, N., \& Dullemond, C. P. 2004, A\&A, 427, 245

Stetson, P. B. 1987, PASP, 99, 191

Teixeira, R., Ducourant, C., Sartori, M. J., et al. 2000, A\&A, 361, 1143

Torres, G., Guenther, E. W., Marschall, L. A., et al. 2003, AJ, 125, 2, 825

Van Leeuwen, F. 2007a, A\&A, 474, 653

Van Leeuwen, F. 2007b, Hipparcos, the New Reduction of the Raw Data, Series: Astrophysics and Space Science Library (Springer), 350

Webb, R. A., Zuckerman, B., Platais, I., et al. 1999, ApJ, 512, L63

Zuckerman, B., \& Song, I. 2004, ARA\&A, 42, 685 